\title{
Prescrire correctement la physiothérapie
}

\author{
De nombreuses réactions du monde médical avant, mais surtout après la \\ parution de l'article «La pression monte - sur les physiothérapeutes et sur \\ les médecins» [1], ont révélé des incertitudes sur la prescription de la physio- \\ thérapie. physioswiss a publié une notice destinée à clarifier cette situation.
}

\section{Annick Kundert}

Vice-présidente du domaine «Santé» de physioswiss

1 Gross B. La pression monte sur les physiothérapeutes et sur les médecins. Bull Med Suisse. 2008;89(23):1016.

Correspondance: Annick Kundert physioswiss

Centralstrasse $8 \mathrm{~b}$ CH-6210 Sursee Tel. 0419266969 Fax 0419266999 info@physioswiss.ch
Le site Internet de physioswiss propose une notice «Prescrire de la physiothérapie - mais comment?» (www.physioswiss.ch ), qui répond aux questions les plus importantes concernant la prescription de physiothérapie. Les incertitudes qui subsistent à ce sujet devraient ainsi être dissipées et le travail administratif réduit. Les points clés de cette notice sont présentés ci-dessous.

Les physiothérapeutes facturent leurs services aux tarifs en vigueur pour la physiothérapie.

Ces tarifs ont été définis dans le cadre d'une convention entre physioswiss et Santésuisse (conformément à la LAMal 46 et à l'OPAS 5), agréée au plan national et par le Conseil fédéral. Ces tarifs sont basés sur des forfaits à la séance et sur des suppléments, selon les cas.

Les médecins de toutes les spécialités peuvent établir des prescriptions de physiothérapie, même les dentistes. Une prescription est valable trois mois à partir de sa date de délivrance. Le médecin peut demander neuf séances au maximum par prescription. Il est possible de prescrire deux traitements par jour, mais le médecin doit le mentionner expressément sur le formulaire.

Pour que les prestations des physiothérapeutes soient remboursées par les assureurs, la prescription doit mentionner un diagnostic médical abrégé ou un code diagnostique, mais un diagnostic global et non spécifique suffit dans la plupart des cas. Pour les diagnostics spécifiques, le médecin ne peut remettre la prescription qu'au médecin-conseil.

Le prescripteur n'est pas tenu de justifier aux assureurs les positions tarifaires choisies par les physiothérapeutes. Cette justification est fournie par les physiothérapeutes en cas de traitements coûteux.

Les assureurs peuvent demander des précisions écrites au médecin lorsque le diagnostic abrégé ou le code de diagnostic ne figurent pas sur la prescription ou lorsqu'un complément d'information est requis (p.ex. pour un cas complexe ou un cas de lymphœdème). Au terme de la phase de rééducation, des traitements de longue durée peuvent être prescrits. Ce n'est que dans ce cas, après quatre séries de traitements (36 traitements), qu'une justification écrite du médecin peut être requise pour un même diagnostic (OPAS 5, art. 4).

Les assureurs-maladie ne peuvent pas limiter le nombre de séances par patient et par an: la LAMal ne prévoit aucune limite par patient et par année civile.

La notice «Prescrire de la physiothérapie mais comment?» est disponible sur le site www.physioswiss.ch $\rightarrow$ Services $\rightarrow$ Downloads et liens. Pour toute question, n'hésitez pas à contacter le secrétariat général de physioswiss au tél. 0419266969. 